\title{
Matrix Completion Discriminant Analysis
}

\author{
Tong Tong $\mathrm{Wu}^{1}$ and Kenneth Lange ${ }^{2}$ \\ Running Title: Matrix Completion Discriminant Analysis
}

\begin{abstract}
${ }^{1}$ Tong Tong $\mathrm{Wu}$ is Associate Professor in the Departments of Biostatistics and Computational Biology, University of Rochester, NY 14642 (email: tongtong_wu@urmc.rochester.edu).

${ }^{2}$ Kenneth Lange is Professor of Biomathematics, Human Genetics, and Statistics at the University of California, Los Angeles, CA 90095 (email: klange@ucla.edu).
\end{abstract}




\begin{abstract}
Matrix completion discriminant analysis (MCDA) is designed for semi-supervised learning where the rate of missingness is high and predictors vastly outnumber cases. MCDA operates by mapping class labels to the vertices of a regular simplex. With $c$ classes, these vertices are arranged on the surface of the unit sphere in $c-1$ dimensional Euclidean space. Because all pairs of vertices are equidistant, the classes are treated symmetrically. To assign unlabeled cases to classes, the data is entered into a large matrix (cases along rows and predictors along columns) that is augmented by vertex coordinates stored in the last $c-1$ columns. Once the matrix is constructed, its missing entries can be filled in by matrix completion. To carry out matrix completion, one minimizes a sum of squares plus a nuclear norm penalty. The simplest solution invokes an MM algorithm and singular value decomposition. Choice of the penalty tuning constant can be achieved by cross validation on randomly withheld case labels. Once the matrix is completed, an unlabeled case is assigned to the class vertex closest to the point deposited in its last $c-1$ columns. A variety of examples drawn from the statistical literature demonstrate that MCDA is competitive on traditional problems and outperforms alternatives on large-scale problems.
\end{abstract}

Key words: Classification; Missing observations; MM algorithm; Semisupervised learning; Singular value decomposition 


\section{Introduction}

Whenever large data sets are collected, missing responses and missing predictors occur. Missing data values are now classified as missing completely at random (MCAR), missing at random (MAR), and missing not at random (MNAR). Statisticians have devised a host of methods for coping with missing data, including: listwise deletion, pairwise deletion, hot deck imputation, mean substitution, regression substitution, and multiple imputation. In model fitting in general, and regression in particular, the modern tendency is to impute missing values by maximum likelihood estimates or posterior means under a Gaussian model (Little and Rubin 2002; Schafer 2010). Regardless of the method of imputation, the consensus among statisticians is that one should use all available information. Failures to impute data can increase bias and compromise inference.

For data presented in matrix form, a new imputation method is now available. Matrix completion aims to recover a full matrix - usually of low rank - from a subset of observed entries. During the past five years, the subject of matrix completion has captured the attention of researchers from a variety of backgrounds in statistics, applied mathematics, and computer science. Candès and Recht (2009) prove that a low-rank matrix can be almost perfectly recovered when the number of observed entries exceeds a certain level. Fortunately, their strong uniformity condition on sampled entries can be relaxed (Recht 2011). Matrix completion can be accomplished by several algorithms: subspace evolution and transfer (SET) (Dai and Milenkovic 2009), gradient algorithms applied to the primal and dual problems (Lin et al. 2009), singular value thresholding (SVT) (Cai et al. 2010; Hu et al. 2012), fixed point and Bregman iteration (Ma et al. 2011), the alternating 
direction method (Chen et al. 2012; Yuan et al. 2009), modified fixed point continuation (Ma and Zhi 2011), and alternating minimization (Jain et al. 2012).

Methods for handling missing data in discriminant analysis have lagged methods for model fitting. Most research has focused on classification trees and the nature of missingness, for example, whether missingness occurs in the testing data (Saar-Tsechansky and Provost 2007), in the training data, or in a combination of both (Ding and Simonoff 2010). Other relevant distinctions include missing responses in both training and testing (Ding and Simonoff 2010) and MCAR (Feelders 1999; Kim and Yates 2003; Zhang et al. 2005) versus a combination of MCAR, MAR, and MNAR (Kalousis and Hilario 2000; Twala 2009). Farhangfar et al. (2008) evaluated a variety of imputation methods on classifiers for discrete data, while Sun et al. (2009) studied the impact of imputation on classification accuracy with DNA microarray data. A common theme among these and other papers (Acuna and Rodriguez 2004; Luengo et al. 2012; Garcia-Laencina et al. 2010) is the deterioration of classification accuracy as the rate of missingness increases. Standard imputation methods are ill equipped to handle high rates of missingness. Two existing papers advocate matrix completion to handle missing data in transductive learning (Cabral et al. 2011; Goldberg et al. 2010). However, these papers deal with multi-label classification rather than multi-category classification and employ a fixed point continuation algorithm rather than an MM algorithm.

Our new combination of matrix completion with Vertex Discriminant Analysis (VDA) (Lange and Wu 2008; Wu and Lange 2010; Wu and Wu 2012) extends VDA into the realm of semi-supervised learning. VDA is geometrically motivated and attuned to matrix representation. Missing ob- 
servations are ubiquitous in practice, and discarding cases with missing predictors leads to less accurate classification. Instead of conducting imputation and classification sequentially, we believe that classification can inform imputation and advocate conducting them simultaneously. Our new method, which we call Matrix Completion Discriminant Analysis (MCDA), is specifically designed for data exhibiting high rates of missingness and an excess of features over cases. It is precisely in this setting of high-dimensional sparse data that matrix completion is expected to shine. Our cancer classification results validate this intuition and justify the inclusion of incomplete cases and the strategy of simultaneous imputation and classification.

\section{Matrix Completion Discriminant Analysis}

\subsection{Vertex Discriminant Analysis}

VDA is a novel supervised classification method (Lange and Wu 2008; Wu and Lange 2010; Wu and Wu 2012). In classification with $c$ classes, it operates by mapping the classes to the $c$ vertices of a regular simplex in the Euclidean space $\mathbb{R}^{c-1}$. For example in binary classification, the two classes correspond to the numbers -1 and 1 on the real line. In trinary classification, the three classes correspond to the three vertices of an equilateral triangle in the plane. The advantages of mapping categories to vertices include dimension reduction, simplification of computation, ease of interpretation, and enhancement of geometric intuition. It is impossible to situate more than $c$ equidistant points in $\mathbb{R}^{c-1}$ (Lange and Wu 2008).

There are several versions of VDA: VDA $\left(\right.$ Lange and Wu 2008), VDA $\mathrm{VE}_{\mathrm{L}}$ (Wu and Lange 2010), and $\mathrm{VDA}_{\mathrm{K}}$ (Wu and $\mathrm{Wu} 2012$ ), where the subscripts stand for Ridge, Lasso and Euclidean, and Kernel, respectively. The first two are linear classifiers, and the third is a nonlinear classifier. The linear VDA 
classifiers rely on the linear regression model $\boldsymbol{y}_{i}=\boldsymbol{A} \boldsymbol{x}_{i}+\boldsymbol{b}, i=1, \ldots, n$, to predict the vertex associated with case $i$. Here $\boldsymbol{x}_{i}$ is a $p$-dimensional predictor vector for case $i, \boldsymbol{A}=\left(a_{j k}\right)$ is a $(c-1) \times p$ matrix of slopes, and $\boldsymbol{b}=\left(b_{j}\right)$ is a $c-1$ column vector of intercepts. The linear VDA classifiers minimized the objective function

$$
R(\boldsymbol{\theta})=\frac{1}{n} \sum_{i=1}^{n} g\left(\boldsymbol{y}_{i}-\boldsymbol{A} \boldsymbol{x}_{i}-\boldsymbol{b}\right)+\lambda P(\boldsymbol{A}),
$$

where $g(\boldsymbol{z})=\|\boldsymbol{z}\|_{2, \epsilon}=\max \left\{\|\boldsymbol{z}\|_{2}-\epsilon, 0\right\}$ denotes $\epsilon$-insensitive Euclidean distance, $\boldsymbol{\theta}$ signifies the parameters $(\boldsymbol{A}, \boldsymbol{b})$, and $P(\boldsymbol{A})$ denotes the penalty imposed on $\boldsymbol{A}$. In $\mathrm{VDA}_{\mathrm{R}}$,

$$
P(\boldsymbol{A})=\sum_{j=1}^{c-1} \sum_{k=1}^{p} a_{j k}^{2}
$$

is a ridge penalty, while in $\mathrm{VDA}_{\mathrm{LE}}$,

$$
P(\boldsymbol{A})=\sum_{j=1}^{c-1} \sum_{k=1}^{p}\left|a_{j k}\right|+\sum_{j=1}^{c-1} \sqrt{\sum_{k=1}^{p} a_{j k}^{2}}
$$

is a mixture of lasso and Euclidean penalties. Lasso and Euclidean penalties promote spareness in the estimate of the slope matrix $\boldsymbol{A}$.

Nonlinear $\mathrm{VDA}_{\mathrm{K}}$ exploits reproducing kernel Hilbert spaces (RKHS). Every such Hilbert space of functions $\mathcal{H}_{K}$ is generated by a kernel function $K(\cdot, \cdot)$. If we fix a point $\boldsymbol{y}$, then the function $\boldsymbol{x} \mapsto K(\boldsymbol{x}, \boldsymbol{y})$ belongs to $\mathcal{H}_{K}$. In the current setting $\boldsymbol{x}$ is the predictor vector that discriminates between the various classes. The inner product of two such functions $\boldsymbol{x} \mapsto K(\boldsymbol{x}, \boldsymbol{y})$ and $\boldsymbol{x} \mapsto K(\boldsymbol{x}, \boldsymbol{z})$ turns out to be $K(\boldsymbol{y}, \boldsymbol{z})$. In $\mathrm{VDA}_{\mathrm{K}}$ we minimize the objective function

$$
\frac{1}{n} \sum_{i=1}^{n} g\left[\boldsymbol{y}_{i}-f\left(\boldsymbol{x}_{i}\right)\right]+\lambda \sum_{j=1}^{c-1}\left\|h_{j}\right\|_{\mathcal{F}_{K}}^{2},
$$


where $f\left(\boldsymbol{x}_{i}\right)$ has $j$ th component

$$
f_{j}(\boldsymbol{x})=h_{j}(\boldsymbol{x})+b_{j}=\sum_{k=1}^{n} a_{j k} K\left(\boldsymbol{x}, \boldsymbol{x}_{k}\right)+b_{j} .
$$

The parameters in the model are again the slopes $a_{j k}$ and intercepts $b_{j}$. Given the bilinearity of the kernel, the objective function can be written as

$$
\frac{1}{n} \sum_{i=1}^{n} g\left[\boldsymbol{y}_{i}-\boldsymbol{f}\left(\boldsymbol{x}_{i}\right)\right]+\lambda \operatorname{tr}\left(\boldsymbol{A} \boldsymbol{K} \boldsymbol{A}^{T}\right),
$$

where $\boldsymbol{K}$ is the $n \times n$ symmetric matrix $\left[K\left(\boldsymbol{x}_{k}, \boldsymbol{x}_{l}\right)\right]$ and $\boldsymbol{A}$ is the $(c-1) \times$ $n$ matrix $\left(a_{j k}\right)$. An attractive feature of $\mathrm{VDA}_{\mathrm{K}}$ is that optimization and estimation are performed on $O(n)$ parameters rather than $O(p)$ parameters, where $n$ is the number of cases, and $p$ is the number of features.

\subsection{Matrix Completion}

Matrix completion (Cai et al. 2010; Candès and Tao 2010; Chen et al. 2012; Mazumder et al. 2010) dispenses with linear regression and approximates a matrix $\boldsymbol{Y}=\left(y_{i j}\right)$ with missing entries by a low-rank matrix $\boldsymbol{X}=\left(x_{i j}\right)$ of the same dimensions. In matrix completion one minimizes the objective function

$$
f(\boldsymbol{X})=\frac{1}{2} \sum_{(i, j) \in \Delta}\left(y_{i j}-x_{i j}\right)^{2}+\lambda \sum_{k} \sigma_{k},
$$

where $\Delta$ collects the index pairs $(i, j)$ of the observed entries of $\boldsymbol{Y}$ and $\sigma_{i}$ is the $i$ th singular value of $\boldsymbol{X}$. Recall that the singular value decomposition (SVD) $\boldsymbol{X}=\sum_{i} \sigma_{i} \boldsymbol{u}_{i} \boldsymbol{v}_{i}^{T}$ of $\boldsymbol{X}$ involves orthonormal singular vectors $\boldsymbol{u}_{i}$ and $\boldsymbol{v}_{i}$ and nonnegative singular values $\sigma_{i}$ presented in descending order. The penalty $\sum_{k} \sigma_{k}$ is the nuclear norm $\|\boldsymbol{X}\|_{\text {nuc }}$ of $\boldsymbol{X}$. It plays a role in low-rank matrix approximation similar to that played by the $\ell_{1}$ norm $\|\boldsymbol{b}\|_{1}=\sum_{k}\left|b_{k}\right|$ 
in sparse regression. The positive tuning constant $\lambda$ controls the strength of the penalization. The objective function (2) can be rewritten as

$$
\frac{1}{2}\left\|P_{\Delta}(\boldsymbol{Y})-P_{\Delta}(\boldsymbol{X})\right\|_{F}^{2}+\lambda\|\boldsymbol{X}\|_{\text {nuc }}
$$

using the Frobenius norm $\|\boldsymbol{X}\|_{F}=\sqrt{\operatorname{tr}\left(\boldsymbol{X} \boldsymbol{X}^{T}\right)}=\sqrt{\sum_{i} \sum_{j} x_{i j}^{2}}$ and the projection operator $P_{\Delta}(\boldsymbol{Y})$ with components

$$
P_{\Delta}(\boldsymbol{Y})_{i j}= \begin{cases}y_{i j} & \text { if }(i, j) \in \Delta \\ 0 & \text { if }(i, j) \notin \Delta .\end{cases}
$$

To recover $\boldsymbol{Y}$, one simply replaces a missing entry $y_{i j}$ by the corresponding entry $x_{i j}$ of $\boldsymbol{X}$; observed entries are left untouched.

\subsection{Matrix Completion Discriminant Analysis}

Discriminant analysis (supervised learning) involves both labeled and unlabeled cases. The labeled cases constitute the training sample. In semisupervised learning, the problem requires (a) imputing missing predictors, (b) building a classification rule from the training sample, and (c) classifying the unlabeled cases. The traditional solution takes these steps sequentially. A wide variety of imputation and supervised classification rules exist. In our numerical comparisons to MCDA, we try a few of the most promising combinations. In contrast, MCDA performs imputation and classification simultaneously. When we map class labels to vertices, we assume that all components of a vertex are present or all components are absent. Each case should have at least one predictor present; otherwise, it is non-informative for classification.

Suppose there are $c$ classes, $p$ predictors, $n_{1}$ labeled cases, and $n_{2}$ unlabeled cases, for a total of $n=n_{1}+n_{2}$ cases. As with other VDA methods, we map the $c$ classes to the $c$ vertices of a regular simplex in $\mathbb{R}^{c-1}$. There 
are an infinity of such simplices. One appealing simplex has vertices

$$
\boldsymbol{v}_{j}= \begin{cases}(c-1)^{-1 / 2} \mathbf{1} & \text { if } j=1 \\ r \mathbf{1}+s \boldsymbol{e}_{j-1} & \text { if } 2 \leq j \leq c\end{cases}
$$

where

$$
r=-\frac{1+\sqrt{c}}{(c-1)^{3 / 2}}, \quad s=\sqrt{\frac{c}{c-1}},
$$

and $\boldsymbol{e}_{j}$ is the $j$ th standard coordinate vector in $\mathbb{R}^{c-1}$. These choices place the vertices on the surface of the unit sphere in $\mathbb{R}^{c-1}$. Any rotation, dilation, or translation of these vectors preserves the equidistant property. This particular geometric encoding of classes has two virtues. First, it is parsimonious compared to mapping classes to the standard unit vectors in $\mathbb{R}^{c}$. Second, it puts the class vertices and standardized predictors on a common unit scale. For a more detailed discussion of the merits of VDA, we refer the reader to our previous VDA papers (Lange and Wu 2008; Wu and Lange 2010; Wu and $\mathrm{Wu} 2012)$.

Without loss of generality, in MCDA we insert the $n$ cases row by row into two blocks of a big matrix $\boldsymbol{U}$, with labeled cases coming above unlabeled cases. The two blocks $\boldsymbol{X}_{1}$ and $\boldsymbol{X}_{2}$ have dimensions $n_{1} \times p$ and $n_{2} \times p$, respectively. To the right of these blocks we insert the vertex coordinates case by case into two blocks $\boldsymbol{V}_{1}$ and $\boldsymbol{V}_{2}$ with dimensions $n_{1} \times(c-1)$ and $n_{2} \times(c-1)$. All of the entries of $\boldsymbol{V}_{1}$ are present, and all of the entries of $\boldsymbol{V}_{2}$ are absent. Overall, the matrix $\boldsymbol{U}$ has the form

$$
\boldsymbol{U}=\left(\begin{array}{ll}
\boldsymbol{X}_{1} & \boldsymbol{V}_{1} \\
\boldsymbol{X}_{2} & \boldsymbol{V}_{2}
\end{array}\right)
$$

We then complete the matrix $\boldsymbol{U}$ to the matrix $\boldsymbol{U}^{*}$ with blocks

$$
\boldsymbol{U}^{*}=\left(\begin{array}{ll}
\boldsymbol{X}_{1}^{*} & \boldsymbol{V}_{1}^{*} \\
\boldsymbol{X}_{2}^{*} & \boldsymbol{V}_{2}^{*}
\end{array}\right)
$$


where $\boldsymbol{V}_{1}^{*}=\boldsymbol{V}_{1}$. The class of an unlabeled case can be assigned by finding the closest vertex to the appropriate row of $\boldsymbol{V}_{2}^{*}$. Because of the shrinkage entailed by the nuclear norm penalty, it is a good idea to standardize all predictors prior to their entry into $\boldsymbol{X}_{1}$ and $\boldsymbol{X}_{2}$. The vertex entries are already scaled properly since class vertices are unit vectors. Because matrix completion is insensitive to row and column permutations of the data, it is simply a matter of convenience to append class vertices on the right of the predictor matrix and to arrange labeled and unlabeled samples in blocks.

\subsection{An MM Algorithm for Matrix Completion}

For the sake of simplicity in the current study, we used the MM algorithm for matrix completion (Mazumder et al. 2010; Lange et al. 2013). See the references (Lange 1995; Hunter and Lange 2004; Lange 2004) for an exposition of the MM principle and numerous specific MM algorithms. Construction of the current MM algorithm is guided by the observation that one can exactly minimize the objective function (2) by a soft-thresholded SVD algorithm when $\boldsymbol{X}$ is complete (Donoho et al. 1995; Mazumder et al. 2010). Suppose $\boldsymbol{U}^{m}$ is the current approximation to $\boldsymbol{U}^{*}$. To restore the symmetry of full data, we replace a missing value $u_{i j}$ by the corresponding entry $u_{i j}^{m}$ of $\boldsymbol{U}^{m}$ and add the term $\left(u_{i j}^{m}-u_{i j}\right)^{2} / 2$ to the objective. This creates a surrogate function that equals the objective at $\boldsymbol{U}^{m}$ and lies above it for all other values of $\boldsymbol{U}$.

One can formalize this construction by introducing the complementary projection $P_{\Delta^{C}}(\boldsymbol{U})$ satisfying $\boldsymbol{U}=P_{\Delta}(\boldsymbol{U})+P_{\Delta^{C}}(\boldsymbol{U})$. Setting

$$
\boldsymbol{V}^{m}=P_{\Delta}(\boldsymbol{U})+P_{\Delta^{C}}\left(\boldsymbol{U}^{m}\right)
$$


allows one to write the surrogate function as

$$
\begin{aligned}
g\left(\boldsymbol{U} \mid \boldsymbol{U}^{m}\right) & =\frac{1}{2}\left\|\boldsymbol{V}^{m}-\boldsymbol{U}\right\|_{F}^{2}+\lambda\|\boldsymbol{U}\|_{\text {nuc }} \\
& =\frac{1}{2}\left\|\boldsymbol{V}^{m}\right\|_{F}^{2}-\operatorname{tr}\left(\boldsymbol{V}^{m} \boldsymbol{U}^{T}\right)+\frac{1}{2}\|\boldsymbol{U}\|_{F}^{2}+\lambda\|\boldsymbol{U}\|_{\text {nuc }}
\end{aligned}
$$

The third term in equation (5) reduces to $\left\|\boldsymbol{U}^{*}\right\|_{F}^{2}=\sum_{k} \sigma_{k}^{2}$. The second term satisfies the von-Neumann-Fan inequality (Borwein and Lewis 2006)

$$
\operatorname{tr}\left(\boldsymbol{V}^{m} \boldsymbol{U}^{T}\right) \leq \sum_{k} w_{k} \sigma_{k}
$$

based on the ordered singular values $w_{k}$ of $\boldsymbol{V}^{m}$ and $\sigma_{k}$ of $\boldsymbol{U}$. Equality is attained when the matrices $\boldsymbol{V}^{m}$ and $\boldsymbol{U}$ have ordered singular value decompositions sharing their left and right singular vectors. In this circumstance, we can rewrite the surrogate function (5) as

$$
\begin{aligned}
g\left(\boldsymbol{U}^{*} \mid \boldsymbol{U}^{m}\right) & =\frac{1}{2} \sum_{k} w_{k}^{2}-\sum_{k} w_{k} \sigma_{k}+\frac{1}{2} \sum_{k} \sigma_{k}^{2}+\lambda \sum_{k} \sigma_{k} \\
& =\frac{1}{2} \sum_{k}\left(w_{k}-\sigma_{k}\right)^{2}+\lambda \sum_{k} \sigma_{k} .
\end{aligned}
$$

It is easy to see that the shrunken singular values $\sigma_{k}=\max \left\{w_{k}-\lambda, 0\right\}$ are optimal. In practice, one only needs to extract the singular values $w_{k}>\lambda$ and corresponding singular vectors to construct the next approximate $\boldsymbol{U}^{m+1}$. This MM algorithm, along with all MM algorithms, is a descent algorithm in the sense that it consistently drives the objective function downhill.

\subsection{Choice of Tuning Constant $\lambda$}

To determine a nearly optimal value for the tuning constant $\lambda$, we employed $K$-fold cross validation on the labeled cases. This involves dividing the data into $K$ equal subsamples, leaving one subsample out per fold, training the classifier based on the remaining data, and computing the testing error on the 
omitted sample. The average testing error $c(\lambda)$ across these $K$ subsamples is recorded and approximately minimized. In principle, one can compute $c(\lambda)$ on a dense grid of points and identify the optimal $\lambda$ on the grid. This approach can be computationally inefficient, so we recommend the simple search strategy of taking an initial $\lambda_{0}$ equal to the largest singular value of $P_{\Delta}(\boldsymbol{U})$ and working downward. One keeps reducing $\lambda$ by multiplying it by a fixed factor $0<r<1$ until $c\left(\lambda_{k+1}\right) \geq c\left(\lambda_{k}\right)$. The optimal value is then set to $\lambda_{k}$.

\subsection{Soft Classification}

So far we have discussed hard classification based on assigning a case to its nearest class vertex. In contrast, soft classification operates by estimating class probabilities and presenting a range of possible classes (Wahba and Wahba 2002; Liu et al. 2011). Liu et al. (2011) have pointed out that hard classifiers tend to work better when the class signal is weak, while soft classifiers tend to work better in high-dimensional settings with $p \gg n$ or when the class signal is strong. In applications such as image and speech recognition or classification of cancer subtypes, soft classifiers are often preferred. Because matrix completion avoids probabilistic modeling, calculation of posterior assignment probabilities is not an option. As an alternative, we suggest computing the Euclidean distances $d_{i j}=\left\|\hat{\boldsymbol{v}}_{i}-\boldsymbol{v}_{j}\right\|$ between the imputed row $\hat{\boldsymbol{v}}_{i}$ of $\boldsymbol{V}_{2}^{*}$ corresponding to case $i$ and the vertex $\boldsymbol{v}_{j}$ corresponding to class $j$. The indices $r_{i j}=1 / d_{i j}$ can then be used to rank the assignment choices for case $i$. In our numerical examples, we will employ hard classification unless noted to the contrary. Misclassification is fairly common in the cancer data sets when the missing rate is high. Fortunately, soft classification sets containing the two nearest class vertices almost invariably capture the correct 
class vertex.

\section{$3 \quad$ Numerical Studies}

In the following numerical examples, we first created the missing data by randomly deleting both features and class labels at prescribed rates via the create.missing function in the $m i$ package of R. Mapping of labels to vertices was performed after deletion. Hence, in the MCDA matrix the block $\boldsymbol{V}_{1}$ had no entries missing, and the block $\boldsymbol{V}_{2}$ had all entries missing. As a semisupervised classifier, MCDA performs imputation and classification over all the samples simultaneously. The cases with deleted class labels were used as testing data.

For comparison to MCDA, we combined three method of imputation with four methods of classification. The imputation methods included missForest (Stekhoven and Buehlmann 2012), mice (van Buuren and GroothuisOudshoorn 2011), and imputePCA (Josse and Husson 2013). All three were employed in our first example; for the sake of brevity only missForest was employed in the remaining examples. In classification we used linear discriminant analysis (LDA), the $k$-nearest neighbor method (KNN) with fixed $k=2$ or optimal $k$ between 1 to 10 , one-versus-rest binary support vector machines (OVR), and random forest prediction (RanFor). We conducted 3 -fold cross-validation to determine the optimal tuning parameters for KNN and MCDA.

\subsection{Zoo Data Analysis}

Our first example compares the classification accuracies of MCDA and the two-step methods on the zoo data (Forsyth 1990) from the UCI machine learning repository (Murphy and Aha 1994). This animal classification prob- 
lem consists of 101 cases spread over 7 categories (mammal, bird, fish, reptile, amphibian, insect, and invertebrate). Each case is classified on 16 features, 15 of which are binary and 1 of which (number of legs) is numeric. We randomly deleted both features and classes at missing rates of $30 \%, 50 \%$, $70 \%$, and $90 \%$. To compare MCDA to competitors, we averaged across 100 different random deletion experiments at each rate.

Table 1 compares the average misclassification rates of MCDA to those of the two-step procedures. Standard errors appear in parentheses in the table, and boldface type emphasizes the lowest misclassification rates. When the missing rate is low to moderate, random forest has the lowest error rates. As the missing rate increases, MCDA's accuracy improves relative to the other methods and displays the lowest error rates. The accuracy of the two-step approach is insensitive to which three imputation method is applied. Since missForest is the fastest, we used it alone to impute the data in step 1 of the subsequent examples.

\subsection{Glass, Wine, and Lympho Data Analysis}

We also compared the performance of MCDA to other classification methods on three other benchmark data sets from the UCI machine learning repository: glass, wine, and lympho. These data sets and the zoo data are overdetermined $(n>p)$. Missing data were created in the same way as in the zoo example. Table 2 summarizes the misclassification rates of MCDA and the competing methods, including now the model-based semi-supervised classification Upclass method (Cribbin 2008). The triples beneath each data set give in order the number of categories $c$, the number of cases $n$, and the number of predictors $p$. On the glass and wine data, MCDA outperforms the other methods when the missing rate is high. At moderate missing rates, the 
Table 1: Comparison of mean (standard error) misclassification rates of MCDA and various imputation-classification methods on the zoo data. Each mean is averaged over 100 replicates at each missing rate.

\begin{tabular}{|c|c|c|c|c|c|}
\hline \multirow[b]{2}{*}{ Imputation } & \multicolumn{5}{|c|}{ Missing Rate } \\
\hline & Classification & $30 \%$ & $50 \%$ & $70 \%$ & $90 \%$ \\
\hline & MCDA & $13.4(0.71)$ & $21.8(0.47)$ & $\overline{\mathbf{3 4 . 5}(0.58)}$ & $\mathbf{6 6 . 1 8 ( 0 . 9 4 )}$ \\
\hline missForest & $\overline{L D A}$ & $10.8(0.55)$ & $23.3(0.61)$ & $\overline{\mathrm{NA}}$ & $\overline{\mathrm{NA}}$ \\
\hline missForest & $\mathrm{KNN}(2)$ & $13.5(0.68)$ & $25.8(0.69)$ & $47.7(0.71)$ & $73.59(0.77)$ \\
\hline missForest & $\mathrm{KNN}$ & $11.8(0.64)$ & $24.5(0.58)$ & $45.4(0.70)$ & $71.54(0.86)$ \\
\hline missForest & OVR & $13.5(0.63)$ & NA & NA & NA \\
\hline missForest & RanFor & $10.4(0.54)$ & $20.9(0.57)$ & $42.6(0.68)$ & $70.40(0.91)$ \\
\hline mice & LDA & $10.8(0.55)$ & $23.3(0.61)$ & NA & NA \\
\hline mice & $\mathrm{KNN}(2)$ & $12.9(0.60)$ & $25.7(0.68)$ & $47.6(0.74)$ & $72.58(0.82)$ \\
\hline mice & $\mathrm{KNN}$ & $12.3(0.62)$ & $24.5(0.64)$ & $45.0(0.74)$ & $71.52(0.90)$ \\
\hline mice & OVR & $13.5(0.63)$ & NA & NA & $\mathrm{NA}$ \\
\hline mice & RanFor & $10.5(0.58)$ & $21.0(0.59)$ & $42.7(0.69)$ & $70.34(0.89)$ \\
\hline imputePCA & LDA & $10.8(0.55)$ & $23.3(0.61)$ & $\mathrm{NA}$ & $\mathrm{NA}$ \\
\hline imputePCA & $\mathrm{KNN}(2)$ & $12.9(0.62)$ & $25.5(0.62)$ & $47.7(0.71)$ & $73.50(0.78)$ \\
\hline imputePCA & KNN & $11.9(0.59)$ & $24.7(0.65)$ & $45.1(0.72)$ & $71.53(0.88)$ \\
\hline imputePCA & OVR & $13.5(0.63)$ & NA & NA & $\mathrm{NA}$ \\
\hline imputePCA & RanFor & $\mathbf{1 0 . 4}(0.59)$ & $20.8(0.59)$ & $42.5(0.70)$ & $70.43(0.92)$ \\
\hline
\end{tabular}


misclassification rates of MCDA are close to the best rates. For the lympho data, MCDA has the lowest misclassification rates at all three missing rates.

\subsection{High-dimensional Cancer Subtype Classification}

In contrast to the small benchmark UCI data sets, we now investigate a few high-dimensional cancer classification problems where $p \gg n$. The data sets colon (Alon et al. 1999), prostate (Singh et al. 2002), SRBCT (Khan et al. 2001), and brain (Pomeroy et al. 2000) allow one to classify the molecular subtypes of these cancers based on a patient's microarray gene expression levels. We randomly deleted observations via create.missing in $m i$ at missing rates 50\%, 70\%, and 90\%. Table 3, which averages over 50 replicates, makes it clear that MCDA's performance is uniformly best. Since matrix completion was designed to recover large-scale matrices, this is the edge we expected.

Because the misclassification rates for these data sets are high, we evaluated soft classification. For example, with the SRBCT data given 50\% missing data, the misclassification rate was $11.7 \%$. Almost all of the misclassified cases are covered by the soft classification set containing the two closest class vertices. Furthermore, the ratio $r_{i j} / r_{i k}$ of the best reciprocal distance to the second best reciprocal distance is always close to 1 for misclassified cases and well in excess of 1 for correctly classified cases. Figure 1 displays a boxplot of this ratio for the correctly and incorrectly classified cases for the SRBCT and brain examples. It would be helpful to establish a cutoff level. For a ratio exceeding the cutoff, the closest-vertex rule would be deemed trustworthy. On the basis of just a handful of data sets, it is probably premature to set a rule of thumb. 
Table 2: Comparison of mean (standard error) misclassification rates of MCDA and other classification methods on the glass, wine, and lympho data. Each mean is averaged over 100 replicates at the missing rates of $50 \%$, $70 \%$, and $90 \%$. Missing entries were imputed by the missForest method. The triples beneath each data set give in order the number of categories $c$, the number of cases $n$, and the number of predictors $p$.

\begin{tabular}{llccc}
\hline Missing & Classification & $\begin{array}{c}\text { Glass } \\
(6,214,10)\end{array}$ & $\begin{array}{c}\text { Wine } \\
(3,178,13)\end{array}$ & $\begin{array}{c}\text { Lympho } \\
(4,148,18)\end{array}$ \\
\hline $50 \%$ & MCDA & $53.75(0.36)$ & $13.39(0.30)$ & $\mathbf{3 5 . 4}(0.59)$ \\
& LDA & $48.0(0.52)$ & $10.8(0.34)$ & NA \\
& KNN(2) & $49.6(0.49)$ & $34.6(0.55)$ & $53.2(2.15)$ \\
& KNN & $45.6(0.52)$ & $31.7(0.53)$ & $51.4(2.32)$ \\
& OVR & NA & $10.2(0.33)$ & NA \\
& RanFor & $\mathbf{4 2 . 1}(0.50)$ & $11.6(0.37)$ & $48.7(2.84)$ \\
& Upclass & $50.2(0.61)$ & $\mathbf{9 . 9}(0.34)$ & $68.8(2.68)$ \\
\hline $70 \%$ & MCDA & $61.60(0.36)$ & $\mathbf{2 3 . 5 4}(0.33)$ & $\mathbf{4 4 . 9 3 ( 0 . 4 4 )}$ \\
& LDA & $58.8(0.43)$ & $25.9(0.42)$ & NA \\
& KNN(2) & $62.2(0.47)$ & $43.9(0.49)$ & $63.3(1.78)$ \\
& KNN & $58.7(0.50)$ & $40.2(0.61)$ & $62.4(2.01)$ \\
& OVR & NA & $24.1(0.42)$ & NA \\
RanFor & $\mathbf{5 7 . 4}(0.44)$ & $25.6(0.46)$ & $62.0(2.36)$ \\
& Upclass & $61.2(0.49)$ & $23.8(0.46)$ & $78.5(1.21)$ \\
\hline MCDA & $\mathbf{6 9 . 0 8}(0.32)$ & $\mathbf{5 3 . 2 4}(0.35)$ & $\mathbf{6 6 . 3 1}(0.48)$ \\
& LDA & NA & $63.36(0.51)$ & NA \\
KNN(2) & $70.66(0.50)$ & $61.97(0.55)$ & $69.15(1.29)$ \\
KNN & $69.68(0.50)$ & $61.61(0.56)$ & $70.33(1.44)$ \\
& OVR & NA & $60.63(0.57)$ & NA \\
RanFor & $69.74(0.52)$ & $59.71(0.64)$ & $69.99(1.51)$ \\
& Upclass & $73.9(0.61)$ & $62.0(0.57)$ & NA \\
\hline & & & & \\
& & & & \\
& & &
\end{tabular}



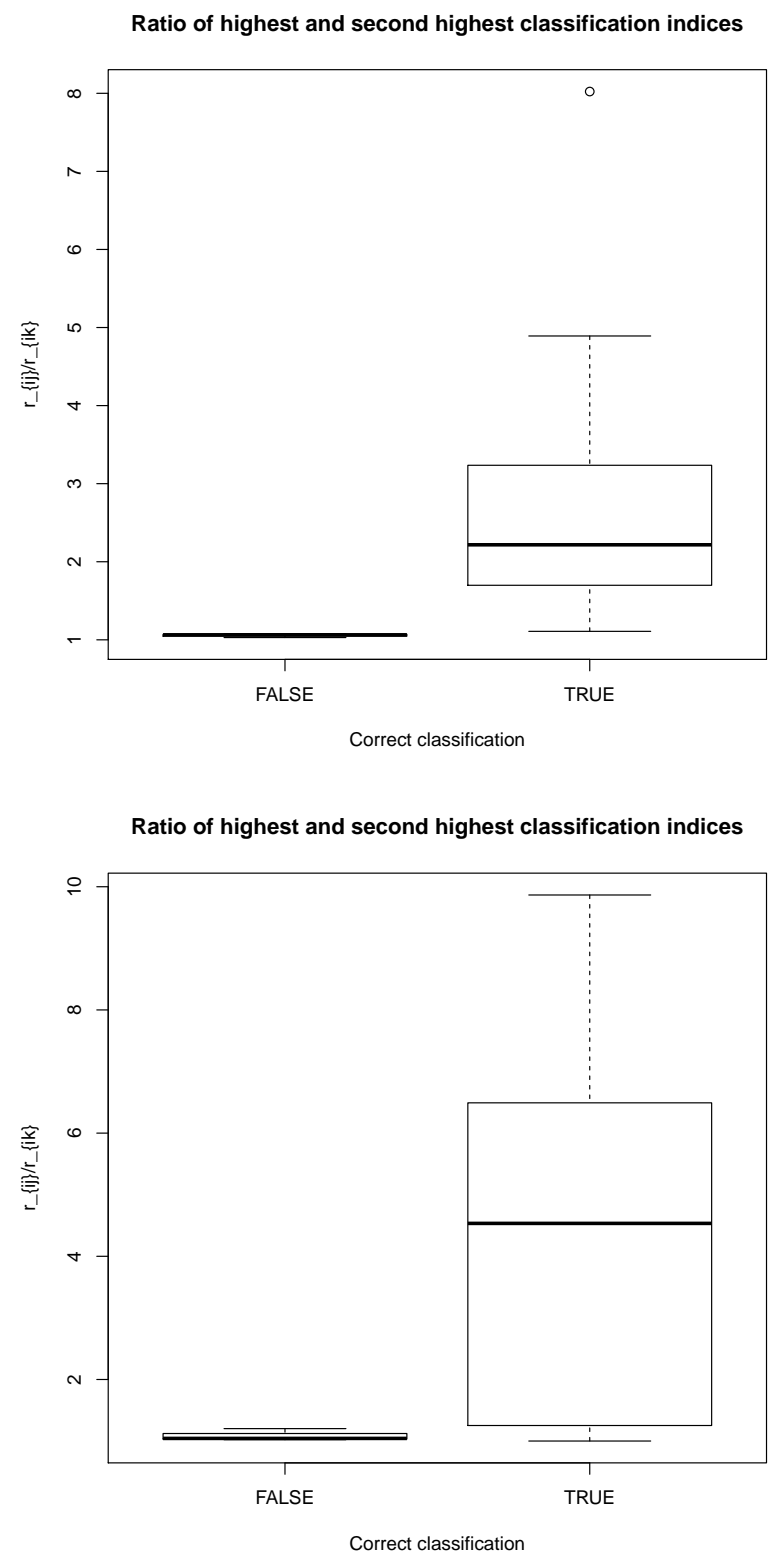

Figure 1: Boxplot of the ratio of the highest classification index $r_{i j}$ to the second highest classification index $r_{i k}$ for the SRBCT (upper panel) and brain (lower panel) data with $50 \%$ missing rate 
Table 3: Comparison of mean (standard error) misclassification rates of MCDA and other classification methods on four benchmark cancer data sets. Each mean is averaged over 50 replicates at the missing rates of $50 \%$, $70 \%$, and $90 \%$. Missing entries were imputed by the missForest method. The triples beneath each data set give in order the number of categories $c$, the number of cases $n$, and the number of predictors $p$.

\begin{tabular}{llcccc}
\hline Missing & Classification & $\begin{array}{c}\text { Colon } \\
(2,62,2000)\end{array}$ & $\begin{array}{c}\text { Prostate } \\
(2,102,6033)\end{array}$ & $\begin{array}{c}\text { SRBCT } \\
(4,63,2308)\end{array}$ & $\begin{array}{c}\text { Brain } \\
(5,42,5597)\end{array}$ \\
\hline $50 \%$ & MCDA & $\mathbf{1 9 . 1 6}(0.65)$ & $\mathbf{1 4 . 4 2}(1.34)$ & $\mathbf{1 1 . 7 0}(0.86)$ & $\mathbf{2 3 . 0 6}(1.25)$ \\
& LDA & $19.69(0.79)$ & $16.90(0.81)$ & $20.26(1.44)$ & NA \\
& KNN $(2)$ & $26.44(0.97)$ & $23.72(0.57)$ & $19.87(1.33)$ & $36.43(1.12)$ \\
& KNN & $25.11(1.00)$ & $20.63(0.67)$ & $21.35(1.69)$ & $30.48(0.86)$ \\
& OVR & $28.00(1.47)$ & $19.89(0.90)$ & $20.26(1.30)$ & NA \\
& RanFor & $21.98(1.06)$ & $17.83(0.92)$ & $18.97(1.33)$ & $39.76(1.67)$ \\
\cline { 2 - 6 } $70 \%$ & MCDA & $\mathbf{2 2 . 8 5}(0.78)$ & $\mathbf{2 1 . 0 9}(1.04)$ & $\mathbf{1 9 . 2 6}(0.71)$ & $\mathbf{2 5 . 4 5 ( 0 . 6 6 )}$ \\
& LDA & $23.77(0.85)$ & $21.87(1.04)$ & $43.37(1.11)$ & NA \\
& KNN $(2)$ & $31.12(0.75)$ & $33.06(0.72)$ & $38.22(1.13)$ & $44.53(1.17)$ \\
& KNN & $27.86(0.85)$ & $33.43(0.88)$ & $35.45(1.98)$ & $41.38(1.64)$ \\
& OVR & $31.54(1.19)$ & $29.13(1.33)$ & $49.09(1.39)$ & NA \\
& RanFor & $26.23(1.08)$ & $21.28(1.20)$ & $35.98(1.17)$ & $45.73(1.27)$ \\
\cline { 2 - 6 } $90 \%$ & MCDA & $\mathbf{3 5 . 1 8}(0.98)$ & $\mathbf{4 1 . 3 3}(1.01)$ & $\mathbf{4 9 . 5 9}(0.77)$ & $\mathbf{5 0 . 5 1}(1.08)$ \\
& LDA & $50.39(1.60)$ & $45.22(1.00)$ & $68.58(1.07)$ & NA \\
& KNN $(2)$ & $51.95(1.29)$ & $47.25(1.11)$ & $67.22(0.94)$ & $75.11(1.44)$ \\
& KNN & $44.94(1.47)$ & $47.97(0.95)$ & $68.49(0.81)$ & $72.40(1.36)$ \\
& OVR & $42.08(1.33)$ & $49.18(0.72)$ & $68.24(0.91)$ & NA \\
& RanFor & $49.87(1.69)$ & $46.59(0.98)$ & $66.92(1.03)$ & $76.53(1.92)$ \\
\hline
\end{tabular}




\subsection{Computational Speed}

Besides better accuracy and easy one-step implementation, MCDA enjoys advantages in numerical stability and computational speed. Table 4 records

the average computing times of MCDA and competing methods on some of the sample data sets. Times include imputation via missForest and identification of tuning constants, but not random deletion of entries. All runs were carried out on a 64-bit Windows 7 desktop computer. MCDA is always faster than the competing methods, especially when conducted by the lowrank matrix fitting (LMAFit) algorithm (Wen et al. 2012; Shen et al. 2014). Savings are more dramatic in underdetermined problems than overdetermined problems and when missing rates are lower. MCDA computing times are subject to further improvements if a faster SVD algorithm is swapped into the MM algorithm, or another matrix completion method is employed.

In general, the MM algorithm is fast, robust, and sure to converge. It is, however, by no means the fastest algorithm for matrix completion. To give the reader a feel for the speed of the MM algorithm versus competing algorithms, we randomly populated the entries of two matrices $\boldsymbol{A}$ and $\boldsymbol{B}$ of dimensions $n_{1} \times r$ and $r \times n_{2}$ with standard normal deviates. We then computed the rank $r$ product $\boldsymbol{M}=\boldsymbol{A} \boldsymbol{B}$, added random deviates with standard deviation 0.001 to its entries, and randomly deleted a prescribed fraction of these perturbed entries. Table 5 displays the number of iterations until convergence and the total computing time for five methods: (a) the MM algorithm, (b) the singular value thresholding (SVT) algorithm in MATLAB (Cai et al. 2010), (c) the fixed point continuation (FPC) algorithm with approximate SVD in MATLAB (Ma et al. 2011), (d) the LMAFit algorithm in MATLAB (Wen et al. 2012; Shen et al. 2014), and (e) the iterative softthresholded svd (softImpute) algorithm in R (Hastie et al. 2014). FPC is 
Table 4: Mean computing time (in seconds) for MCDA and other classification methods. Times include missForest imputations on the wine and glass data. Missing rates are $50 \%$ and $90 \%$.

\begin{tabular}{llcccc}
\hline Missing & Classification & Wine & Glass & Colon & SRBCT \\
\hline $50 \%$ & MCDA & 0.15 & 0.17 & 92.9 & 132.6 \\
& MCDA+LMaFit & 0.07 & 0.05 & 0.12 & 0.14 \\
& missForest+LDA & 2.41 & 2.15 & 3317.4 & 4159.4 \\
& missForest+KNN(2) & 2.40 & 2.14 & 3316.0 & 4157.8 \\
& missForest+KNN & 2.41 & 2.14 & 3316.1 & 4157.9 \\
& missForest+OVR & 2.43 & NA & 30431.5 & 3316.6 \\
& missForest+RanFor & 2.47 & 2.23 & 3316.8 & 4158.9 \\
\cline { 2 - 5 } $90 \%$ & MCDA & 0.37 & 0.29 & 170.2 & 213.1 \\
& MCDA+LMaFit & 0.08 & 0.09 & 0.18 & 0.21 \\
& missForest+LDA & 0.45 & NA & 3895.0 & 5403.4 \\
& missForest+KNN(2) & 0.44 & 0.35 & 3894.0 & 5402.4 \\
& missForest+KNN & 0.45 & 0.35 & 3894.0 & 5402.4 \\
& missForest+OVR & 0.46 & NA & 3894.5 & 5181.9 \\
& missForest+RanFor & 0.46 & 0.66 & 3894.1 & 5181.2 \\
\hline
\end{tabular}

less reliable compared to other algorithms and fails to converge if the standard deviation of the added noise exceeds 0.005. Examination of the table suggests that the MM algorithm is competitive if the tuning constant is well chosen.

Other contenders for fast matrix completion are mentioned in the literature. For example, the rank-one matrix update rule for SVD algorithms (Wang et al. 2014) and the ADMM algorithm for bi-linear decomposition (Glashoff and Bronstein 2012) could be more efficient than the algorithms tested here. From our vantage point, superior discrimination is more important than sheer speed. 
Table 5: Computing time (in seconds) for the MM and other matrix completion algorithms

\begin{tabular}{cccrr}
\hline Size $\left(n_{1} \times n_{2}\right)$ & Rank $r$ & Algorithm & \# Iters & Time \\
\hline $150 \times 300$ & 10 & MM & 14 & 11.9 \\
& & SVT & 500 & 30.6 \\
& FPC & 111 & 15.5 \\
& LMaFit & 28 & 0.06 \\
\multirow{3}{*}{$150 \times 300$} & \multirow{2}{*}{50} & softImpute & 128 & 0.89 \\
\cline { 3 - 5 } & & MM & 6 & 4.5 \\
& SVT & 500 & 47.1 \\
& FPC & 2230 & 330.9 \\
& & LMaFit & 13 & 0.08 \\
$1000 \times 1000$ & \multirow{3}{*}{10} & softImpute & 34 & 0.22 \\
\cline { 3 - 5 } & & MM & 2 & 1.78 \\
& SVT & 500 & 57.8 \\
& & FPC & 323 & 1714.5 \\
& & LMaFit & 41 & 0.48 \\
& & softImpute & 243 & 35.99 \\
\cline { 3 - 5 } $1000 \times 1000$ & \multirow{2}{*}{100} & MM & 7 & 5.78 \\
& & SVT & 59 & 92.2 \\
& & FPC & NA & NA \\
& & LMaFit & 8 & 0.57 \\
& & softImpute & 71 & 8.86 \\
\hline & & &
\end{tabular}




\section{Discussion}

Our examples demonstrate the promise of MCDA in discriminant analysis with missing data. In ordinary discriminant analysis it would be hard to improve on established methods, and we make no claim to this effect. Given the ubiquity of missing data and the prevalence of big data in modern statistics, MCDA has the potential to improve inference. It is competitive on small data sets with low missing rates. Its edge in extreme circumstances likely stems from (a) combining the steps of imputation and classification, (b) encoding classes in a geometrically symmetric and parsimonious way, and (c) filling in missing entries by matrix completion. Matrix completion does entail shrinkage, but this should not distort classification much. The rule of assigning an unlabeled case to the closest class vertex works fine if shrinkage is spherically symmetric and keeps imputed points within the conical zones of assignment of the class vertices.

The rigorous assumptions under which matrix completion have been validated most likely can be relaxed. Our examples feature completely random deletion of predictors and case labels. Deletion of vertex coordinates is an all or nothing decision and obviously not completely random. We have applied matrix completion to SNP (single nucleotide polymorphism) imputation in genetics with considerable success despite highly nonrandom patterns of missingness (Chi et al. 2013). For example, individuals are often genotyped on different but overlapping SNP sets, creating a strongly structured missingness pattern in a study's genotype matrix.

MCDA is fast and capable of handling large data sets. The MM algorithm is conceptually simple and numerically stable. Its speed can be improved by limiting its extraction of singular values and vectors to those 
that enter the imputed matrix. Other algorithms may prove faster on large data sets, so we are inclined to keep an open mind about tweaking the computational engine. The biggest drawback of MCDA is that handling a new case requires rerunning the entire analysis. All unlabeled cases, including a new one, inform discrimination.

Establishing the theoretical correctness of MCDA will be a challenge. To our frustration we have not been able to prove Fisher consistency, the minimal theoretical requirement of a classification method. A loss function $L(\boldsymbol{y}, \boldsymbol{x})$ is said to be Fisher consistent if minimizing its average risk $\mathrm{E}\{L[f(\boldsymbol{X}), \boldsymbol{Y})]\}$ under the assignment rule $f(\boldsymbol{x})$ leads to the optimal Bayes decision rule. Tackling the consistency of MCDA is difficult because it relies on a complicated penalty, cross validation, and murky rules for missing entries. In developing MCDA our intuitive reach has exceeded our rigorous grasp. We would be happy for help from other statisticians in resolving the thorny questions of theory.

Of greater urgency from our perspective is the development of new applications of MCDA. One potential application that comes to mind is medical diagnosis. Suppose we consider the 100 most common ailments. Patients are scored on many tests of blood chemistry, physiology, imaging, and family history. Given the expense of testing, most patients are scored on a small minority of the possible tests. Diagnostic data is highly structured, and unfortunately, missingness is hardly random. Yet good classification could point physicians in productive directions. Given the uncertainty of medical diagnosis, it probably would be a mistake to make hard class assignments. However, singling out a few of the closest class vertices could inform judgments about what further tests to order. Obviously, a fairly large database of patient histories would be necessary to carry out this research agenda. 


\section{Acknowledgements}

The authors thank Kevin Keys for his help with MATLAB and the reviewers for their constructive comments and suggestions. This research was supported by NSF grant CCF-0926181 and NIH grants from the National Human Genome Research Institute (HG006139) and the National Institute of General Medical Sciences (GM053275).

\section{References}

Acuna, E. and Rodriguez, C. (2004), "The treatment of missing values and its effect in the classifier accuracy," Classification, Clustering and Data Mining Applications, 639-648.

Alon, U., Barkai, N., Notterman, D., Gish, K., Mack, S., and Levine, J. (1999), "Broad patterns of gene expression revealed by clustering analysis of tumor and normal colon tissues probed by oligonucleotide arrays," Proc. Natl. Acad. Sci. USA, 96, 6745-6750.

Borwein, J. M. and Lewis, A. S. (2006), Convex Analysis and Nonlinear Optimization: Theory and Examples, CMS Books in Mathematics, New York: Springer, 2nd ed.

Cabral, R. S., la Torre, F. D., Costeira, J. P., and Bernardino, A. (2011), "Matrix Completion for Multi-label Image Classification." in NIPS, eds. Shawe-Taylor, J., Zemel, R. S., Bartlett, P. L., Pereira, F. C. N., and Weinberger, K. Q., pp. 190-198.

Cai, J.-F., Candès, E. J., and Shen, Z. (2010), "A Singular Value Thresholding Algorithm for Matrix Completion," SIAM J. on Optimization, 20, $1956-1982$. 
Candès, E. J. and Recht, B. (2009), "Exact Matrix Completion via Convex Optimization," Foundations on Computational Mathematics, 9(6), 717772 .

Candès, E. J. and Tao, T. (2010), "The power of convex relaxation: nearoptimal matrix completion," IEEE Trans. Inf. Theor., 56, 2053-2080.

Chen, C., He, B., and Yuan, X. (2012), "Matrix completion via an alternating direction method," IMA Journal of Numerical Analysis, 32, 227-245.

Chi, E. C., Zhou, H., Chen, G. K., Del Vecchyo, D. O., and Lange, K. (2013), "Genotype imputation via matrix completion," Genome Research, $23,509-518$.

Cribbin, L. (2008), "upclass: R Package for Performing Updated Classification Rules," Master's thesis, University College Dublin.

Dai, W. and Milenkovic, O. (2009), "SET: an algorithm for consistent matrix completion," CoRR, abs/0909.2705.

Ding, Y. and Simonoff, J. S. (2010), "An Investigation of Missing Data Methods for Classification Trees Applied to Binary Response Data," J. Mach. Learn. Res., 11, 131-170.

Donoho, D. L., Johnstone, I. M., Kerkyacharian, G., and Picard, D. (1995), "Wavelet shrinkage: asymptopia," Journal of the Royal Statistical Society, Ser. B, 371-394.

Farhangfar, A., Kurgan, L., and Dy, J. (2008), "Impact of imputation of missing values on classification error for discrete data," Pattern Recogn., 41, 3692-3705. 
Feelders, A. J. (1999), "Handling Missing Data in Trees: Surrogate Splits or Statistical Imputation," in Principles of Data Mining and Knowledge Discovery, Third European Conference, PKDD 99, Prague, Czech Republic, September 15-18, 1999, Proceedings, eds. Zytkow, J. M. and Rauch, J., Springer, vol. 1704 of Lecture Notes in Computer Science, pp. 329-334.

Forsyth, R. S. (1990), "PC/BEAGLE User's Guide," .

Garcia-Laencina, P. J., Sancho-Gomez, J.-L., and Figueiras-Vidal, A. R. (2010), "Pattern classification with missing data: a review," Neural Comput. Appl., 19, 263-282.

Glashoff, K. and Bronstein, M. M. (2012), "Structure from Motion Using Augmented Lagrangian Robust Factorization," 2011 International Conference on 3D Imaging, Modeling, Processing, Visualization and Transmission, 379-386.

Goldberg, A. B., Zhu, X., Recht, B., Xu, J.-M., and Nowak, R. D. (2010), "Transduction with Matrix Completion: Three Birds with One Stone." in NIPS, eds. Lafferty, J. D., Williams, C. K. I., Shawe-Taylor, J., Zemel, R. S., and Culotta, A., Curran Associates, Inc., pp. 757-765.

Hastie, T., Mazumder, R., Lee, J., and Zadeh, R. (2014), "Matrix Completion and Low-Rank SVD via Fast Alternating Least Squares," .

Hu, Y., Zhang, D., Liu, J., Ye, J., and He, X. (2012), "Accelerated singular value thresholding for matrix completion," in Proceedings of the 18th ACM SIGKDD international conference on Knowledge discovery and data mining, New York, NY, USA: ACM, KDD '12, pp. 298-306.

Hunter, D. R. and Lange, K. (2004), "A tutorial on MM algorithms," American Statistician, 58, 30-37. 
Jain, P., Netrapalli, P., and Sanghavi, S. (2012), "Low-rank Matrix Completion using Alternating Minimization," CoRR, abs/1212.0467.

Josse, J. and Husson, F. (2013), "Handling missing values in exploratory multivariate data analysis methods," Journal de la SFdS, 153, 79-99.

Kalousis, A. and Hilario, M. (2000), "Supervised knowledge discovery from incomplete data," in Proceedings of the 2nd International Conference on Data Mining 2000, WIT Press.

Khan, J., Wei, J. S., Markus, R., Saal, L. H., Ladanyi, M., Westermann, F., Berthold, F., Schwab, M., Antonescu, C. R., Peterson, C., and Meltzer, P. S. (2001), "Classification and diagnostic prediction of cancers using gene expression profiling and artificial neural networks," Nature Medicine, 7, 673-679.

Kim, H. and Yates, S. (2003), "Missing value algorithms in decision trees," in Statistical Data Mining and Knowledge Discovery, ed. Bozdogan, H., Chapman \& Hall/CRC, pp. 155-172.

Lange, K. (1995), "A gradient algorithm locally equivalent to the EM algorithm," Journal of the Royal Statistical Society, Series B, 57, 425-437.

- (2004), Optimization, New York: Springer-Verlag.

Lange, K., Papp, J. C., Sinsheimer, J. S., and Sobel, E. M. (2013), "Next Generation Statistical Genetics: Modeling, Penalization, and Optimization in High-Dimensional Data," .

Lange, K. and Wu, T. T. (2008), "An MM algorithm for multicategory vertex discriminant analysis," J. Computational and Graphical Statistics, $17,527-544$. 
Lin, Z., Ganesh, A., Wright, J., Wu, L., Chen, M., and Ma, Y. (2009), "Fast convex optimization algorithms for exact recovery of a corrupted low-rank matrix," in In Intl. Workshop on Comp. Adv. in Multi-Sensor Adapt. Processing, Aruba, Dutch Antilles.

Little, R. and Rubin, D. (2002), Statistical analysis with missing data, Wiley series in probability and mathematical statistics. Probability and mathematical statistics, Wiley.

Liu, Y., Zhang, H. H., and Wu, Y. (2011), "Hard or Soft Classification? Large-Margin Unified Machines," Journal of the American Statistical Association, 106, 166-177.

Luengo, J., Garcia, S., and Herrera, F. (2012), "On the choice of the best imputation methods for missing values considering three groups of classification methods," Knowl. Inf. Syst., 32, 77-108.

Ma, S., Goldfarb, D., and Chen, L. (2011), "Fixed point and Bregman iterative methods for matrix rank minimization," Math. Program., 128, 321353.

Ma, Y. and Zhi, L. (2011), "The minimum-rank gram matrix completion via modified fixed point continuation method." in ISSAC, eds. Schost, r. and Emiris, I. Z., ACM, pp. 241-248.

Mazumder, R., Hastie, T., and Tibshirani, R. (2010), "Spectral regularization algorithms for learning large incomplete matrices," Journal of $\mathrm{Ma}$ chine Learning Research, 11, 2287-2322.

Murphy, P. M. and Aha, D. W. (1994), "UCI Repository of machine learning databases [http://www.ics.uci.edu/ mlearn/MLRepository.html]," . 
Pomeroy, S. L., Tamayo, P., Gaasenbeek, M., Sturla, L. M., Angelo, M., McLaughlin, M. E., Kim, J. Y. H., Goumnerova, L. C., Black, P. M., Lau, C., Allen, J. C., Zagzag, D., Olson, J. M., Curran, T., Wetmore, C., Biegel, J. A., Poggio, T., Mukherjee, S., Rifkin, R., Califano, A., Stolovitzky, G., Louis, D. N., Mesirov, J. P., Lander, E. S., and Golub, T. R. (2000), "Molecular portraits of human breast tumours," Nature, 406, 747-752.

Recht, B. (2011), "A Simpler Approach to Matrix Completion," J. Mach. Learn. Res., 12, 3413-3430.

Saar-Tsechansky, M. and Provost, F. (2007), "Handling Missing Values when Applying Classification Models," J. Mach. Learn. Res., 8, 1623-1657.

Schafer, J. (2010), Analysis of Incomplete Multivariate Data, Chapman \& Hall/CRC Monographs on Statistics \& Applied Probability, Taylor \& Francis.

Shen, Y., Wen, Z., and Zhang, Y. (2014), "Augmented Lagrangian Alternating Direction Method for Matrix Separation Based on Low-rank Factorization," Optimization Methods Software, 29, 239-263.

Singh, D., Febbo, P., Ross, K., Jackson, D. G., Manola, J., Ladd, C., Tamayo, P., Renshaw, A. A., DAmico, A. V., Richie, J. P., Lander, E. S., Loda, M., Kantoff, P. W., Golub, T. R., and Sellers, W. R. (2002), "Gene expression correlates of clinical prostate cancer behavior," Cancer Cell, 1, 203-209.

Stekhoven, D. and Buehlmann, P. (2012), "MissForest - nonparametric missing value imputation for mixed-type data," Bioinformatics, 28, 112-118.

Sun, Y., Braga-Neto, U., and Dougherty, E. R. (2009), "Impact of missing value imputation on classification for DNA microarray gene expres- 
sion data: a model-based study," EURASIP J. Bioinformatics Syst. Biol., $2009,4: 1-4: 1$.

Twala, B. (2009), "An Empirical Comparison of Techniques for Handling Incomplete Data Using Decision Trees." Applied Artificial Intelligence, $23,373-405$.

van Buuren, S. and Groothuis-Oudshoorn, K. (2011), "MICE: Multivariate Imputation by Chained Equations in R," Journal of Statistical Software, $45,1-67$.

Wahba, G. and Wahba, G. (2002), "Soft and Hard Classification by Reproducing Kernel Hilbert Space Methods," in Department of Statistics, University of Wisconsin, Madison WI, pp. 16524-16530.

Wang, Z., Lai, M.-J., Lu, Z., Fan, W., Davulcu, H., and Ye, J. (2014), “Orthogonal Rank-One Matrix Pursuit for Low Rank Matrix Completion," arXiv, 1404.1377.

Wen, Z., Yin, W., and Zhang, Y. (2012), "Solving a low-rank factorization model for matrix completion by a nonlinear successive over-relaxation algorithm," Math. Program. Comput., 4(4), 333-361.

Wu, T. T. and Lange, K. (2010), "Multicategory vertex discriminant analysis for high-dimensional data," Ann. Appl. Statist., 4, 1698-1721.

Wu, T. T. and Wu, Y. (2012), "Nonlinear Vertex Discriminant Analysis with Reproducing Kernels," Statistical Analysis and Data Mining, 5, 167-176.

Yuan, M., Joseph, R., and Zou, H. (2009), "Structured Variable Selection and Estimation," Ann. Appl. Stat., in press. 
Zhang, S., Qin, Z., Ling, C. X., and Sheng, S. (2005), "'Missing Is Useful': Missing Values in Cost-Sensitive Decision Trees." IEEE Trans. Knowl. Data Eng., 17, 1689-1693. 